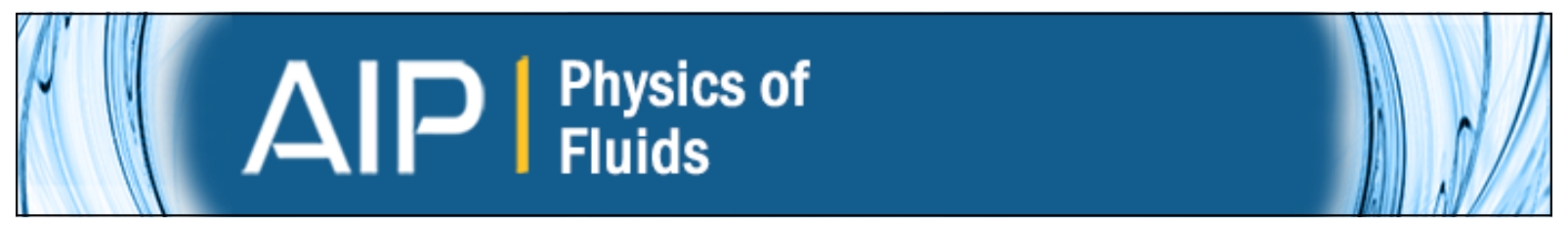

Implications of hydrophobic interactions and consequent apparent slip phenomenon on the entrance region transport of liquids through microchannels

Suman Chakraborty and Kumar Dinkar Anand

Citation: Physics of Fluids (1994-present) 20, 043602 (2008); doi: 10.1063/1.2904988

View online: http://dx.doi.org/10.1063/1.2904988

View Table of Contents: http://scitation.aip.org/content/aip/journal/pof2/20/4?ver=pdfcov

Published by the AIP Publishing

Advertisement:

AlP Re-register for Table of Content Alerts

Create a profile.

Sign up today! 


\title{
Implications of hydrophobic interactions and consequent apparent slip phenomenon on the entrance region transport of liquids through microchannels
}

\author{
Suman Chakraborty a) and Kumar Dinkar Anand \\ Department of Mechanical Engineering, Indian Institute of Technology, Kharagpur 721302, India
}

(Received 11 September 2007; accepted 4 March 2008; published online 22 April 2008)

\begin{abstract}
The implications of entrance region transport in hydrophobic microchannels are theoretically and experimentally investigated in this work. Detailed analytical solutions are derived, depicting the dependences of the liquid phase velocity profiles, entrance lengths, and friction factor variations on the relative thickness of a nanobubble-dispersed layer formed in the vicinity of the microchannel wall as a consequence of localized hydrophobic interactions. It is revealed that even for a layer of nanobubbles formed with a typical thickness in the tune of three orders of magnitude lower than the characteristic microchannel dimensions, the entrance length can be enhanced to the limit of about 1.5 times than that for the cases devoid of any hydrophobic interactions. The pressure drop characteristics in the entrance region, as obtained for such cases, can turn out to be of significant consequence with regard to the design of typical pressure-driven microflow systems involving hydrophobic substrates. Closed-form expressions for the effective friction factor are also derived so that more accurate and scientific guidelines can be provided for design of hydrophobic microchannels, rather than trivially overruling the consequences of entrance region transport that is commonly exercised on a routine basis. (C) 2008 American Institute of Physics.
\end{abstract}

[DOI: $10.1063 / 1.2904988$ ]

\section{INTRODUCTION}

Rapid advancements in microfabrication and miniaturization techniques have resulted in growing demands for employing microfluidic devices and systems in several practical applications of emerging interest, encompassing the areas of biotechnology and biomedical engineering, electronic chip cooling, and inkjet printing, to name a few. Dramatically enhanced significance of interfacial phenomena, originated as a consequence of the drastic reduction in length scales accompanying miniaturization, is perhaps one of the most important facets that dictate the most critical aspects of these applications. While experimental evidences clearly support distinct and nontrivial frictional characteristics of microscale flows as compared to their macroscale counterparts and significant deviations from classical conjectures in many of the concerned devices and systems, the underlying theoretical explanations as well as laboratory observations have often been found to be inconsistent and contradictory.

Despite intense investigations being undertaken on microfluidics and microscale transport processes by several groups of researchers, there has only been a little work done on the issues of entrance region flows in microchannels. As such, researchers have routinely presumed the microchannel flows to be fully developed, ignoring the entrance effects. This has perhaps been based on the characteristic low values of Reynolds numbers characterizing typical microflows. However, it has been revealed that in the entrance region of microchannels, velocity distribution and skin friction charac-

\footnotetext{
a) Author to whom correspondence should be addressed. Electronic mail: suman@mech.iitkgp.ernet.in.
}

teristics may exhibit significant variations in the streamwise direction, ${ }^{1}$ which is likely to bear significant consequences with regard to the efficiencies of microchannel-based separation processes. The entrance effects are not only expected to be a strong function of the channel length, aspect ratio, and the Reynolds number but also are likely to be critically dependent on the interfacial transport at the microchannel walls. The shorter the channels and the larger their aspect ratios, the more significant can these effects be.

Yang $e t \mathrm{al}^{2}$ were among the pioneering groups of researchers to numerically simulate the flow physics in the entrance region of a microchannel, by considering an electroosmotically driven transport. Studies on similar aspects were also reported by Chen et $a l .^{3}$ These investigations, however, were primarily focused on the electro-osmotic flow actuation mechanisms. Campbell and Kandlikar ${ }^{4}$ investigated the effects of entrance conditions on pressure gradients and flow transitions in pressure-driven liquid transport through microchannels and minichannels. None of the above-mentioned studies, however, considered the possibilities hydrophobic interactions and the apparent slip phenomena at the fluidsolid interface and their pertinent consequences on the entrance region transport.

For several decades, investigations on fluid dynamics relied on the assumption that when a liquid flows over a solid surface, there is a zero relative velocity between the liquid molecules and the solid boundary at their points of contact. Although this no-slip boundary condition has been successfully applied to model most of the macroscopic experiments on liquid flows, there has been no microscopic justification behind this conjecture. It is important to mention in this con- 
text that one of the most interesting and important aspects of deviation from the classical no-slip boundary condition in case microchannel liquid flows can be attributed to hydrophobic interactions at the solid-fluid interface, which may be triggered by surface heterogeneities and roughness elements. Accordingly, enhanced flow rates of Newtonian liquids in microchannels are often ascribed to the presence of small amounts of gas trapped on rough and/or hydrophobic surfaces. ${ }^{5,6}$ Considering this hypothesis, Tretheway and Meinhart ${ }^{7}$ devised a predictive methodology for examining the mechanism of measured "apparent" slip behavior of water flowing over hydrophobic microchannel surfaces. By modeling the presence of either a depleted water layer or nanobubbles as an effective gas gap at the microchannel walls, the above authors calculated slip lengths for flow in parallel plate microchannels in their pioneering investigation. Their results clearly suggest that the apparent fluid slip experimentally observed in microchannels with hydrophobic surfaces may arise from either the presence of nanobubbles or a layer of low density fluid at the surface..$^{8-11}$

Motivated by the intriguing influences of the consequent apparent slip phenomenon, researchers have been in a continuous endeavor to understand the detailed mechanism of nanobubble formation in micro-nanofluidic conduits. It is often argued that the interfacial shear mechanisms may induce nucleation of vapor bubbles. ${ }^{12}$ Once the nucleation barrier exceeded, the bubbles grow to cover the surface, and flow of liquid is over this thin gas film rather than the solid surface itself. It is well known that these bubble formations are strongly dependent on surface heterogeneities, roughness, hydrophobicity, as well as gas supersaturation. ${ }^{13}$ Preexisting gas cavities might also play an important role in this context, in the sense that once gas cavities exist on a substrate surface, bubble formation can initiate at reasonably low levels of gas supersaturation. At certain sites on the solid surface, gas may first be trapped, in which case further diffusion of dissolved gas from the bulk solution would enhance the formation of bubbles. ${ }^{14}$ In general, the probability of bubble formation near the interface is likely to be enhanced in the vicinity of a hydrophobic surface. This is primarily because of the fact that the structure of water molecules next to a hydrophobic surface is apparently less ordered than a bulk phase, whereas cohesive strength of water may be significantly reduced with a hydrophobic solid interface. Tyrell and Attard ${ }^{15}$ imaged, with an atomic force microscope, a hydrophobic glass surface submerged in water and discovered the presence of pancake shaped, $20-30 \mathrm{~nm}$ thick nanobubbles completely covering the surface. In addition, they showed that the hydrophobic surface acts as a nucleation site pulling dissolved gasses out of the solution. Within 10-20 min after scraping the surface clean, the surface was once again completely covered with nanobubbles. The idea of slip flow over nanobubble layers was first mentioned by Cottin-Bizonne et al., ${ }^{16}$ as a possible explanation for the attraction between hydrophobic surfaces in water. This attraction could be due to the hydrodynamic correlated fluctuations of the gas interfaces, analogous to the Bjerknes force between two pulsating bubbles. Theoretical considerations have shown that it would be favorable for water between two hydrophobic surfaces to vaporize $^{17}$ although detailed quantification of this phenomenon is yet to be generalized.

Despite substantial advancements in the understanding of microfluidic and nanofluidic systems in recent times, the physics of nanobubble formation, introduced as above, is yet to be fundamentally resolved. ${ }^{18,19}$ Nevertheless, it has been well established that the inception of nanobubble layers in the substrate-liquid interfacial region triggers a sharp discontinuity in the velocity gradients close to the fluid-solid interface. This, in turn, is expected to influence the fully developed velocity profiles to a significant extent, as observed by other researchers. ${ }^{7}$ Such considerations, however, are yet to be extended for analyzing the entrance region transport of liquids in microchannels, with possible nontrivial interactions between the augmented flow resistance due to the entrance effects and reduced flow resistances on account of apparent slip phenomena at the microchannel walls. The resulting fluid dynamic interactions originating as a consequence of these counteracting mechanisms might appear to be somewhat intuitive in nature but are by no means obvious.

The aim of the present work is to devise an analytical formalism for assessing implications of the hydrophobic interaction-induced apparent slip phenomena at the microchannel walls on the pertinent entrance region liquid flow characteristics. Two most significant aspects of the present study are the considerations of effective boundary slip in liquid flows occurring in the entrance region of microchannels and the analytical framework developed for investigating the underlying consequences. Besides their archival implications, the analytical solutions derived in the present study can also be employed to assess and benchmark the accuracy, convergence, and effectiveness of the simplified numerical computation strategies that are commonly adopted for analyzing microscale liquid flows. Further implications of the present analytical solutions lie in the fact that fullscale computational studies alone cannot supply with enough information regarding the significant characteristics of the solution and the comprehensive effect of the entrance region transport since a limited range of data set can only be covered during the numerical experiments and prohibitively refined grid systems are often necessary to capture the nearwall flow features. Thus, irrespective of routinely executed computational studies on fluid dynamic transport over disparate length scales, closed-form solutions on microchannel flows greatly attract the research community, primarily attributable to the following two important aspects: (i) the characteristics of the whole solution can be grasped easily and (ii) bottlenecks related to the requirements of stringent spatiotemporal resolution are of no consequence for analytical solutions. Therefore, the closed-form solutions presented in this study may act as an efficient design basis for microchannels subjected to strong hydrophobic interactions at the walls, especially in cases of "short" microchannels for which the entrance region spans over a significant fraction of the overall axial extent. Utilizing these solutions, the friction factors in the entrance and the fully developed flow regimes are also obtained as a function of the relative thicknesses of the nanobubble layers, depicting the implications of the apparent slip mechanisms on the microchannel flow resistance 
characteristics. Experimental verifications in support of the important findings of the present study are also outlined in this work.

\section{MATHEMATICAL MODELING}

We consider the pressure-driven flow in the hydrodynamic entrance region of a parallel plate microchannel, subjected to hydrophobic interactions at the walls. The microchannel height is taken as $2 h$, with symmetry about $y=0$ (centerline). The momentum equation can be written in a conservative form as (where $x$ represents the axial coordinate and $y$ represents the transverse coordinate)

$$
\frac{\partial u^{2}}{\partial x}+\frac{\partial(u v)}{\partial y}=-\frac{1}{\rho} \frac{d p}{d x}+\nu \frac{\partial^{2} u}{\partial y^{2}},
$$

where the pressure gradient $d p / d x$ is assumed to be independent of $y$. In Eq. (1), $u$ and $v$ are the velocity components along $x$ and $y$ directions, respectively, $\rho$ is the density of the fluid, and $\nu$ is its kinematic viscosity. Equation (1) can be integrated from $y=0$ to $y=h$, to yield

$$
-\frac{1}{\rho} \frac{d p}{d x}=\frac{1}{h} \frac{\partial}{\partial x} \int_{0}^{h} u^{2} d y-\frac{\nu}{h}\left(\frac{\partial u}{\partial y}\right)_{y=h} .
$$

By eliminating $d p / d x$ from Eqs. (1) and (2) and noting that $\partial / \partial x\left(\int_{0}^{h} u d y\right)=0$ (from the continuity equation), one obtains the following equations as a linearization approximation: ${ }^{17}$

$$
\nu \frac{\partial^{2} u}{\partial y^{2}}=\bar{u} \frac{\partial u}{\partial x}+\frac{\nu}{h}\left(\frac{\partial u}{\partial y}\right)_{y=h},
$$

where $\bar{u}$ is the cross-sectionally averaged axial velocity component. Introducing dimensionless variables (considering $D_{h}$ as the hydraulic diameter of the channel) $\xi$ $=\left(x / D_{h}\right) /\left(\bar{u} D_{h} / \nu\right), \eta=y / h$, and $U=u / \bar{u}$, Eq. (3) becomes

$$
\frac{\partial^{2} U}{\partial \eta^{2}}=\frac{1}{16} \frac{\partial U}{\partial \xi}+\left(\frac{\partial U}{\partial \eta}\right)_{\eta=1} .
$$

In solving Eq. (4), it is important to take into account the hydrophobic nature of the microchannel substrate because of which the solid boundaries may not be in direct contact with the bulk liquid. Instead, a thin layer of gas bubbles is likely to mediate the microchannel wall and the transported liquid, as mentioned earlier. With the formation of such vapor bubbles flow over those is likely to result in an apparent slip. $^{20,21}$ Since stress must be continuous at a liquid-gas interface, a difference of shear viscosities will lead to a difference of strain rates. If a liquid of viscosity $\mu_{w}$ flows over a layer of height $\delta$ with viscosity $\mu_{a}$, the apparent slip length for the flow conditions described as above can be estimated as $^{7}$

$$
\beta=\frac{\mu_{w}}{\mu_{a}}\left(\frac{\delta^{2}}{2 h}+\delta\right) .
$$

By utilizing Eq. (5), the appropriate wall boundary condition for Eq. (4) can be written as

$$
U(1, \xi)=-\beta^{\prime}(\partial U / \partial \eta)_{\eta=1},
$$

where $\beta^{\prime}=\beta / h$. The other pertinent boundary conditions are as follows:

$$
\begin{aligned}
& \left(\frac{\partial U}{\partial \eta}\right)_{\eta=0}=0, \\
& U(\eta, 0)=1 .
\end{aligned}
$$

Further, since the flow is fully developed at a "large" distance from the inlet, one may write

$$
U(\eta, \infty)=U_{\mathrm{fd}}(\eta),
$$

where $U_{\mathrm{fd}}(\eta)$ represents the fully developed velocity profile.

In solving Eq. (4), the velocity field can be conceived as a combined consequence of the fully developed and the developing flow profiles in the following manner:

$$
U(\eta, \xi)=U_{\mathrm{fd}}(\eta)+V(\eta, \xi),
$$

where $V(\eta, \xi)$ represents the developing flow velocity profile. Substituting Eq. (10) into Eq. (4), we find that $U_{\mathrm{fd}}(\eta)$ satisfies the following equation:

$$
\frac{d^{2} U_{\mathrm{fd}}}{d \eta^{2}}=\left(\frac{d U_{\mathrm{fd}}}{d \eta}\right)_{\eta=1} .
$$

A solution to Eq. (16) may be obtained by using Eqs. (6) and (7) and the following continuity condition:

$$
\int_{0}^{1} U_{\mathrm{fd}}(\eta) d \eta=1
$$

to yield

$$
U_{\mathrm{fd}}=\frac{6 \beta^{\prime}}{6 \beta^{\prime}+2}-\frac{3\left(1-\eta^{2}\right)}{6 \beta^{\prime}+2} .
$$

Further, substituting Eq. (10) into Eq. (4), $V(\eta, \xi)$ is found to satisfy the following equation:

$$
\frac{\partial^{2} V}{\partial \eta^{2}}=\frac{1}{16} \frac{\partial V}{\partial \xi}+\left(\frac{\partial V}{\partial \eta}\right)_{\eta=1} .
$$

Equation (13) may be solved by employing the separation of variables method, as

$$
V(\eta, \xi)=F(\xi) G(\eta)
$$

Substituting Eq. (14) into Eq. (13), one obtains

$$
\begin{aligned}
& F_{i}(\xi)=C_{i} \exp \left(-16 \alpha_{i}^{2} \xi\right), \\
& \frac{d^{2} G_{i}}{d \eta^{2}}+\alpha_{i}^{2} G_{i}=\left(\frac{d G_{i}}{d \eta}\right)_{\eta=1},
\end{aligned}
$$

where $\alpha_{i}$ are the eigenvalues. A particular solution of Eq. (16) is of the following form:

$$
G_{i}^{p}(\eta)=\frac{1}{\alpha_{i}^{2}}\left(\frac{d G_{i}}{d \eta}\right)_{\eta=1} .
$$

Solution of the homogeneous part of Eq. (16) is of the following form: 


$$
G_{i}^{h}(\eta)=A_{i} \cos \left(\alpha_{i} \eta\right)+B_{i} \sin \left(\alpha_{i} \eta\right) .
$$

By using Eq. (7), it follows that

$$
B_{i}=0 .
$$

Hence, the complete solution of Eq. (16) is of the form

$G_{i}(\eta)=G_{i}^{p}(\eta)+G_{i}^{h}(\eta)=A_{i} \cos \left(\alpha_{i} \eta\right)+\frac{1}{\alpha_{i}^{2}}\left(\frac{d G_{i}}{d \eta}\right)_{\eta=1}$.

Thus, $\left(d G_{i} / d \eta\right)_{\eta=1}=-\alpha_{i} A_{i} \sin \left(\alpha_{i}\right)$ so that one gets

$$
G_{i}(\eta)=\frac{A_{i}}{\alpha_{i}}\left[\alpha_{i} \cos \left(\alpha_{i} \eta\right)-\sin \left(\alpha_{i}\right)\right] .
$$

By using Eq. (6), the eigenvalues $\left(\alpha_{i}\right)$ are found to satisfy the following constraint:

$$
\tan \alpha_{i}=\frac{\alpha_{i}}{1+\beta^{\prime} \alpha_{i}^{2}} .
$$

Further, the functions $G_{i}$ can be normalized in terms of the orthogonality conditions $\left(\int_{-1}^{1} G_{i}^{2} d \eta=1\right)$ to yield

$$
A_{i}=\frac{1}{\sin \left(\alpha_{i}\right)}\left[1+3 \beta^{\prime}+\alpha_{i}^{2} \beta^{\prime 2}\right]^{-1 / 2}
$$

By applying the entrance condition [Eq. (19)], it follows

$$
V(\eta, 0)=1-U_{\mathrm{fd}}(\eta)=\sum_{i=1}^{\infty} C_{i} G_{i}(\eta) .
$$

By using the orthonormality properties of $G_{i}$, the coefficients $C_{i}$ can be obtained in the following manner:

$$
C_{i}=\int_{-1}^{1}\left[1-U_{\mathrm{fd}}(\eta)\right] G_{i}(\eta) d \eta .
$$

By using Eqs. (12) and (21), the above integral may be obtained as

$$
C_{i}=\frac{2 A_{i} \sin \left(\alpha_{i}\right)}{\alpha_{i}} .
$$

By using Eqs. (12), (14), (15), (21), (23), and (26), the velocity profile can be obtained from Eq. (10) as

$$
\begin{aligned}
V(\eta, \xi)= & \frac{6 \beta^{\prime}}{6 \beta^{\prime}+2}+\frac{3\left(1-\eta^{2}\right)}{6 \beta^{\prime}+2} \\
& +\sum_{i=1}^{\infty} \frac{2\left[\alpha_{i} \cos \left(\alpha_{i} \eta\right)-\sin \left(\alpha_{i}\right)\right] \exp \left(-16 \alpha_{i}^{2} \xi\right)}{\alpha_{i}^{2} \sin \left(\alpha_{i}\right)\left[1+3 \beta^{\prime}+\alpha_{i}^{2} \beta^{\prime 2}\right]} .
\end{aligned}
$$

In obtaining the pressure drop, Eq. (2) may be rewritten as

$$
-\frac{1}{2\left(\frac{1}{2} \rho \bar{u}^{2}\right)} \frac{d p}{d \xi}=\frac{\partial}{\partial \xi} \int_{0}^{1} U^{2}(\eta, \xi) d \eta-16\left(\frac{\partial U}{\partial \eta}\right)_{\eta=1} .
$$

For fully developed flow conditions, the above equation simplifies to

$$
\frac{1}{2\left(\frac{1}{2} \rho \bar{u}^{2}\right)} \frac{d p}{d \xi}=16\left(\frac{\partial U_{\mathrm{fd}}}{\partial \eta}\right)_{\eta=1} .
$$

Equation (29) is integrated between 0 and $\xi$ yielding

$$
\frac{[p(0)-p(\xi)]_{\mathrm{fd}}}{\frac{1}{2} \rho \bar{u}^{2}}=-32 \int_{0}^{\xi}\left(\frac{\partial U_{\mathrm{fd}}}{\partial \eta}\right)_{\eta=1}, \quad d \xi=\frac{96 \xi}{1+3 \beta^{\prime}} .
$$

For the general case of developing flows, Eq. (30) assumes a more general form of

$$
\frac{p(0)-p(\xi)}{\frac{1}{2} \rho \bar{u}^{2}}=\frac{96 \xi}{1+3 \beta^{\prime}}+K(\xi) .
$$

By integrating Eq. (28) between 0 and $\xi$ and comparing the outcome with Eq. (31), one gets

$$
\begin{aligned}
K(\xi)= & \frac{0.8}{\left(1+3 \beta^{\prime}\right)^{2}} \\
& -4 \sum_{i=1}^{\infty} \frac{\left[3-\exp \left(-16 \alpha_{i}^{2} \xi\right)\right] \exp \left(-16 \alpha_{i}^{2} \xi\right)}{\alpha_{i}^{2}\left[1+3 \beta^{\prime}+\beta^{\prime 2} \alpha_{i}^{2}\right]} .
\end{aligned}
$$

By substituting Eq. (32) into Eq. (31), the product of friction factor and Reynolds number can be obtained as

$$
\begin{aligned}
f \operatorname{Re}_{D_{h}}= & \frac{24}{1+3 \beta^{\prime}}+\frac{1}{5 \xi\left(1+3 \beta^{\prime}\right)^{2}} \\
& -\sum_{i=1}^{\infty} \frac{\left[3-\exp \left(-16 \alpha_{i}^{2} \xi\right)\right] \exp \left(-16 \alpha_{i}^{2} \xi\right)}{\alpha_{i}^{2} \xi\left(1+3 \beta^{\prime}+\alpha_{i}^{2} \beta^{\prime 2}\right)} .
\end{aligned}
$$

\section{RESULTS AND DISCUSSIONS}

\section{A. Theoretical estimates}

Figure 1 depicts typical velocity profiles in different sections of the microchannel, for various nanobubble layer thicknesses. In the same figure, the velocity profiles obtained from comprehensive numerical simulations (without invoking any linearization approximation) are also shown. The numerical solutions are obtained by solving the coupled continuity and momentum equations in a two-dimensional framework, by employing a pressure-based finite volume method. Pressure-velocity coupling is accomplished by using the SIMPLER algorithm. ${ }^{22}$ The spatial derivatives in the diffusion terms are discretized by central difference scheme. A variable size grid system is considered and variations in the size of grid are smoothly affected, with more densely spaced grids being skewed close to the solid boundaries. The discretized system of linear algebraic equations is numerically solved by employing the line by line tridiagonal matrix algorithm. Convergence criterion for the maximum relative errors in all the discretized equations is set to $10^{-6}$.

From Fig. 1, excellent agreements between the present analytical solutions and the full-scale numerical solutions are apparent. This aptly demonstrates the applicability of the simplifying assumptions made in formulating the present theoretical model for characterizing developing liquid microflows in presence of perceptible hydrophobic interactions. Further, it is evident from Fig. 1 that as the ratio $\delta / h$ increases, difference between the centerline velocity (i.e., $U$ at $\eta=0)$ and the wall-slip velocity (i.e., $U$ at $\eta=1$ ) progressively diminishes at any given axial location. Higher values of $\delta / h$, in effect, reduce the near-wall velocity gradients to a considerable extent. On the other hand, higher values of $\delta / h$ 


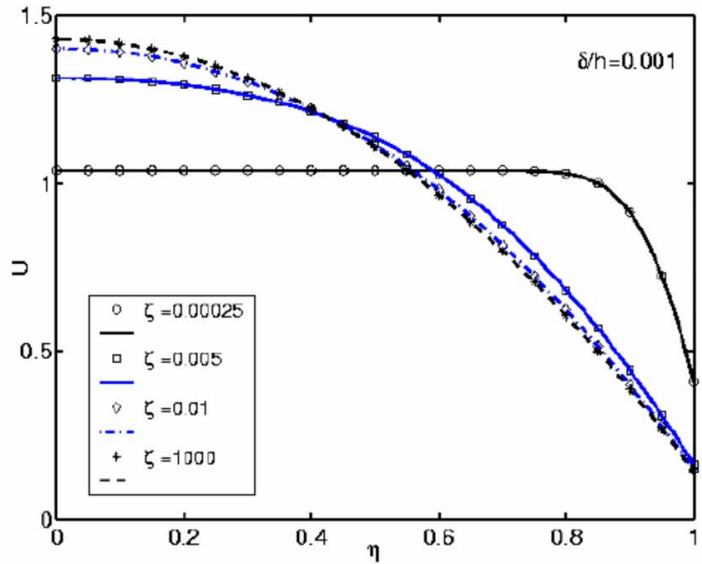

(a)

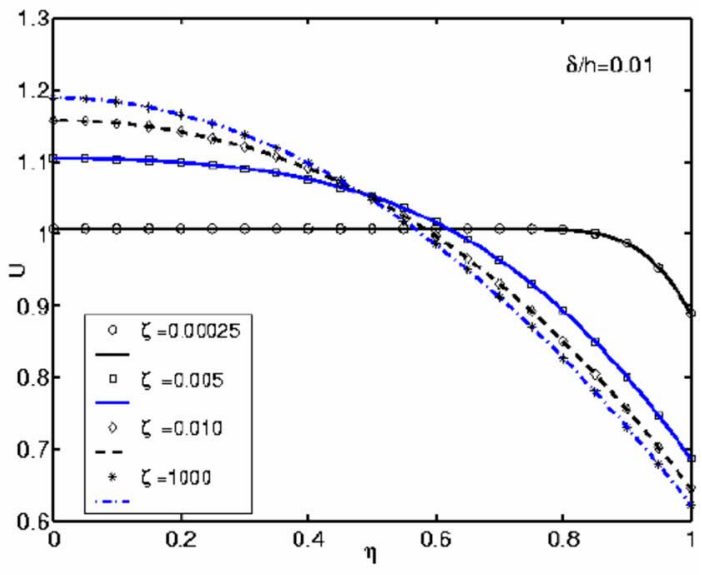

(b)

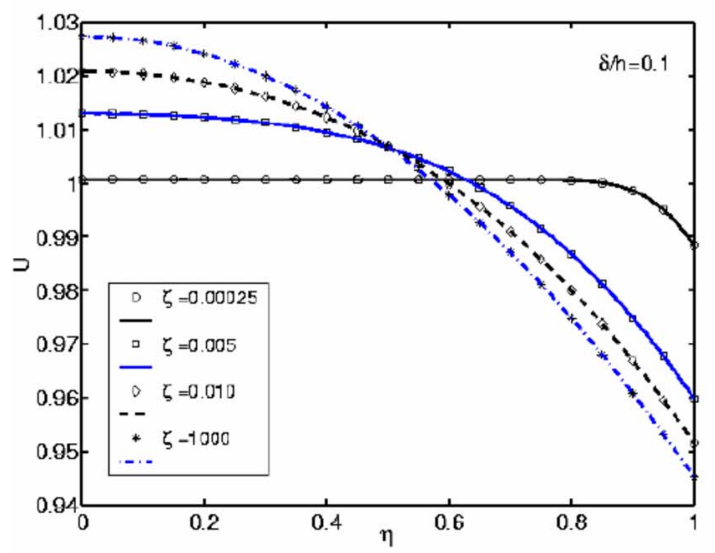

(c)

FIG. 1. (Color online) Cross-sectional velocity profiles at different axial sections corresponding to (a) $\delta / H=0.001$, (b) $\delta / H=0.01$, and (c) $\delta / H$ $=0.1$. Markers in the figures represent the analytical solutions and the continuous lines represent full-scale numerical solutions.

are also attributed to more prominent apparent slip effects due to near-wall hydrophobic interactions. To compensate for that, the apparent slip velocities at the wall turn out to be substantially higher for greater values of $\delta / h$. This effect is

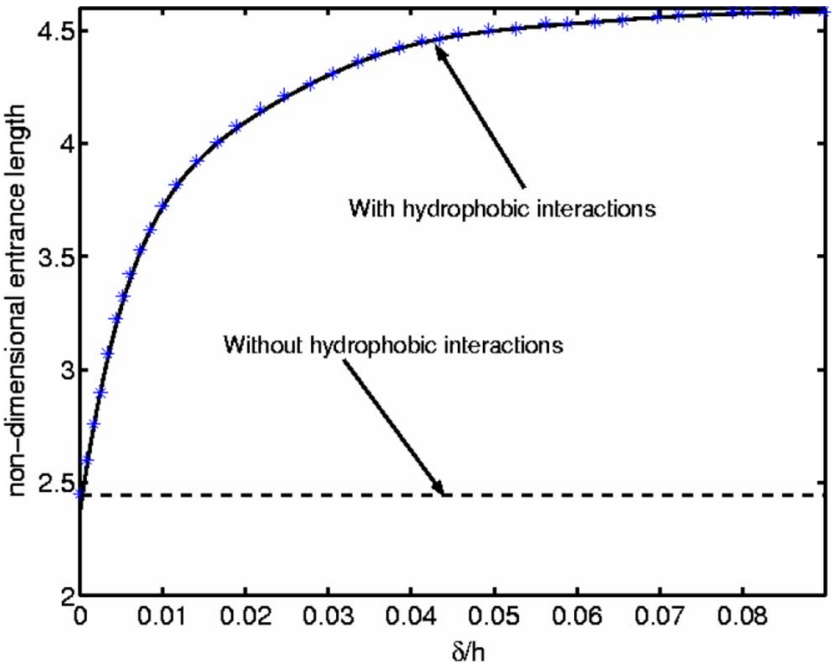

FIG. 2. (Color online) Variations in entrance length with different values of $\delta / H$. Markers in the figure represent the analytical solutions and the continuous lines represent full-scale numerical solutions.

significantly more prominent at locations close to the microchannel entrance. As one axially progresses toward a fully developed state, fluid in the core region tends to accelerate further so as to satisfy the requirements of continuity. This relative acceleration of the central core, however, is considerably reduced for higher values of $\delta / h$, primarily because of the fact that the apparent wall-slippage effects for such cases appear to be strong enough to partly compensate for retarded fluid motion within the viscous shear layer. However, this forces the inner shear layer to grow at a slower rate than the cases without involving any wall-adjacent hydrophobic interactions. This effect is clearly evident in Fig. 2, which depicts the variation of the nondimensional entrance length as a function of $\delta / h$. It is extremely important and interesting to observe from this figure that even with a trace amount of nanobubble formation due to hydrophobic interactions, there is a rapid increment in the nondimensional entrance length (expressed in terms of the parameter $\zeta$ ). In fact, more than $50 \%$ increment in the entrance length can be observed with $\delta / h$ as small as $10^{-5} \%$. This is because of the fact that due to the presence of nanobubble layers blanketing the solid boundaries, the effect of wall shear penetrates into the bulk fluid only after the core flow has axially traversed a significantly greater distance than for the case devoid of any hydrophobic interactions. In more physical terms, an explanation to the above phenomenon can be provided in light of the fact that while the microscopic roughness of the solid surface impede the motion of the adhering fluid promoting the stick flow, the incipient vapor layers formed on the solid surface tend to augment the level of slippage. The vapor layer, in effect, acts like a shield, preventing the liquid from being directly exposed to the surface irregularities. This is very much reminiscent to the consequences of a "superhydrophobicity" phenomenon, as detailed by de Gennes et $a l^{23}$ and Bico and co-workers. ${ }^{24-27}$ In such cases, the liquid is not likely to directly feel the presence of the wall and may smoothly sail over the intervening vapor layers instead of being in direct contact with the wall roughness elements. 


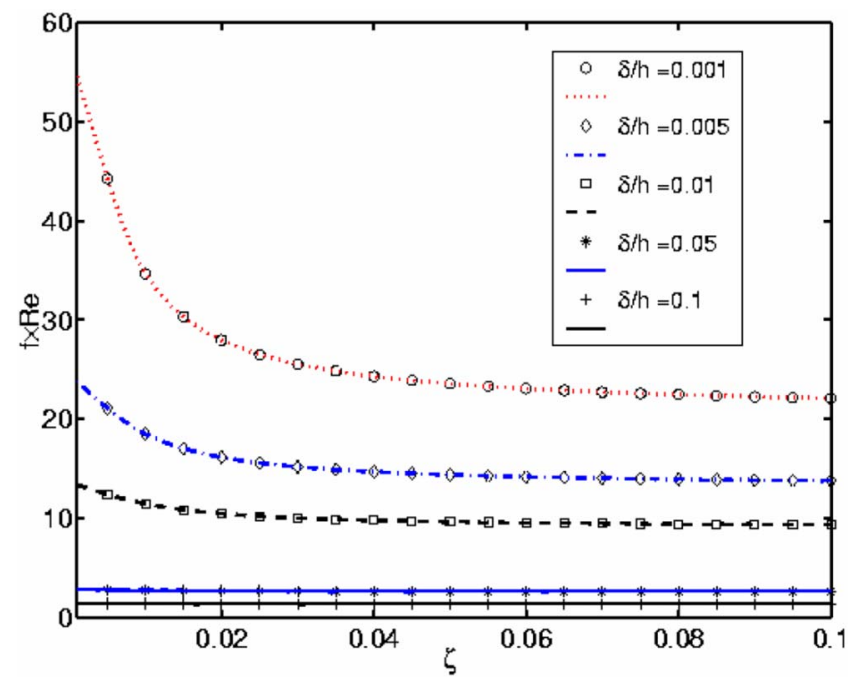

FIG. 3. (Color online) Variations in friction factor for different values of $\delta / H$. Markers in the figures represent the analytical solutions and the continuous lines represent full-scale numerical solutions.

Thus, the apparent slip effects at the wall are primarily responsible for the enhancements in the entrance lengths. Interestingly, the rate of this increment gets somewhat arrested as the value of $\delta / h$ approaches 0.02 . Beyond that limit, the evolution of entrance length with increments in $\delta / h$ is rather asymptotic in nature. Fundamentally, the competing action of two counteracting mechanisms operating in tandem is responsible for this physical behavior. As the nanobubble layer becomes thicker, the apparent slip velocity at the wall tends to increase, which attempts to reduce the local shear layer thickness. On the other hand, this also reduces the normal velocity gradient at the wall, which tends to increase the local shear layer thickness. When these two opposing effects become of comparable strength, no further appreciable enhancement in the entrance length may become possible even with stronger hydrophobic interactions at the wall.

Figure 3 depicts a visual representation of the effective friction factor for different relative thicknesses of the nanobubble layer. Because of an effective slip of the liquid over the nanobubble layer, instead of being confronted with surface roughness elements, the effective friction factor is predicted to be substantially lower as compared to the cases with less prominent hydrophobic interactions. It is interesting to observe in this respect that as the nanobubble layer grows in thickness, marginal differences in friction factor are encountered between the entrance and the fully developed regime. This is because of the significantly weakened convective component of the fluid acceleration along the axial direction in the entrance region with thicker nanobubble layers, which is responsible for a substantially reduced pressure drop than what is intuitively expected for developing flows. With thinner nanobubble layers, however, significantly enhanced frictional pressure drops are encountered in the entrance region, very much reminiscent to the classical developing flow situations.

\section{B. Experimental comparisons}

In order to experimentally assess the physical implications of the present theoretical proposition, rectangular microchannels were fabricated by micromilling cutters of different sizes on $1 \mathrm{~cm}$ thick Perspex ${ }^{\mathrm{TM}}$ glass. During the fabrication procedure, the milling cutters were revolved at a fixed rate of $550 \mathrm{rpm}$ but the feed rates were varied as 12 , 15 , and $20 \mathrm{~mm} / \mathrm{min}$ so as to obtain a range of surface characteristics. ${ }^{28}$ Fluid flow through the microchannels was actuated by means of a peristaltic pump (Amersham Biosciences, Peristaltic Pump P-1, maximum flow rate of $-5000 \mathrm{ml} / \mathrm{h}$ ). For pressure drop measurements across the length of the channel, several pressure tappings were drilled and connected to a pressure sensor (Honeywell, 24 PC series, $0-1 \mathrm{psi}$ ). For illustrating the characteristics of the near wall flow through microchannels, a technique similar to the streaking method for flow visualization ${ }^{29}$ was employed. Briefly, an inverted fluorescent microscope (Olympus inverted fluorescent microscope IX51) coupled with a high speed and high resolution digital camera (4 MPixel Monochrome CCD, ORCA ER, Hamamatsu, Melville, NY, USA) was focused just above the bottom surface of microchannel close to one of the side walls. With $40 \times$ lens, the depth of field was estimated to be $1 \mu \mathrm{m}$. Fluorescent isothiocyanate tagged latex beads (Sigma, USA) of diameter $46 \pm 6 \mathrm{~nm}$ were used as the tracer particles. Over 200 snapshots were taken at the same microscopic field for every sample, with each shot spanning $100 \mathrm{~ms}$ and the images were collected by IMAGE-PRO PLUS software (Media Cybernetics, Bethesda, USA). Subsequently, the out of focus fluorescents, determined from the cut off brightness, were subtracted from original images. The resultant images were overlapped and occupancy of the tracer bead at each pixelated coordinate was estimated. Contrasting intensity and brightness conditions were obtained in the foreground of illuminated fluorescent beads. In the constructed images, a gray upper part highlighted the bulk flow regime, whereas a dark lower part delineated the solid wall. A cloudlike faint intermediate region, proximal to the solid-liquid interface, represented the nanobubble-dispersed intermediate layer. A dilution of bead density at these locations conformed to the imagery depiction of dilution of water molecules in the regions occupied by nanobubbles. Such images effectively provided a direct inference on the occupancy of the microchannel surfaces with the vapor layer of a measurable average thickness.

In obtaining the friction factor as a function of a local axial coordinate, the following expression is employed:

$$
f=\frac{D_{h}}{L} \frac{\Delta p}{\frac{1}{2} \rho \bar{u}^{2}},
$$

where $D_{h}$ is hydraulic diameter of the microchannel, $\Delta p$ is the pressure drop measured across two pressure tappings that are separated by a distance of $L$ along the microchannel axis (one of the tappings being connected to the inlet section and the other tapping being connected to the desired axial location so that the concerned pressure drop represents a local friction factor pertinent to that specific axial coordinate), and $\bar{u}$ is the average velocity of flow (i.e., the ratio of volumetric 
flow rate to the area of cross section). Measurements are thus taken at several axial locations so that local friction factor as a function of the nondimensional axial coordinate may be evaluated. Further details of the pressure drop measurements are outlined in Ref. 28 and are not repeated here for the sake of brevity.

The friction factor values, as obtained from the above experiments, are compared to the corresponding theoretical predictions, as depicted in Fig. 4. These predictions agree quite well each other, thereby establishing the validity of the present physical conjecture.

\section{Toward further generalization}

Results obtained in the present study may further be extended to estimate the entrance region friction factors for microconduits of other shapes. Such estimations may turn out to be critical toward a physically consistent engineering design of microfluidic systems being subjected to substantial hydrophobic interactions. Toward this, an identical theoretical exercise (that has earlier been executed for rectangular shaped channel sections) is first extended to circular sections, by utilizing the governing fluid flow equations in cylindrical coordinate systems. Because of notional analogies between these derivations and the derivations for rectangular sections presented earlier, the detailed derivations for circular sections are not repeated here for the sake of brevity. Rather, direct comparisons between the key results for these two sectional shapes are summarized in Table I for completeness.

Based on the findings of Muzychka and Yovanovich, ${ }^{30}$ which showed that the square root of the cross-sectional area

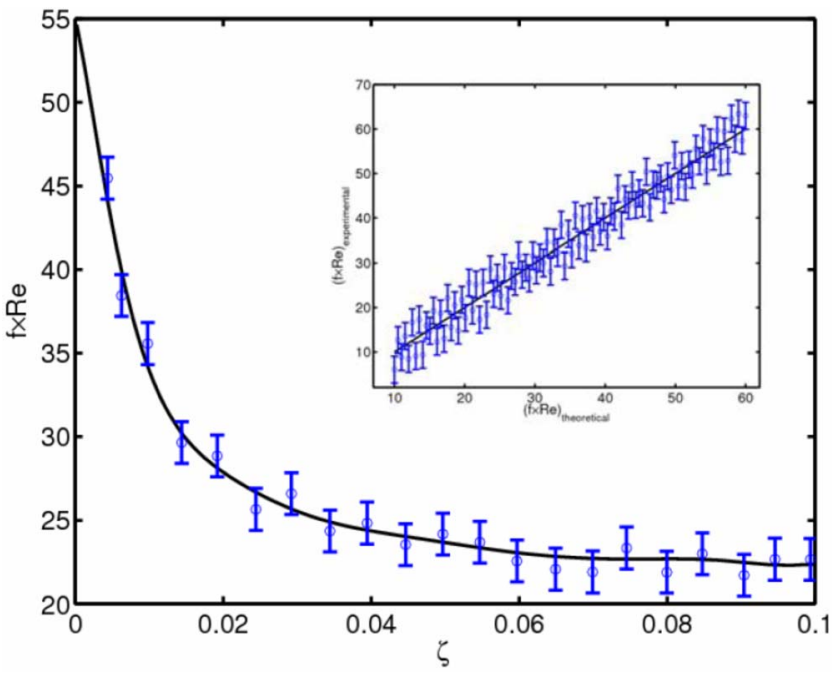

FIG. 4. (Color online) Comparisons between theoretical and experimental predictions of the friction factor, as a function of the nondimensional axial coordinate, for average $\delta / H=0.001$. Markers in the figures represent the experimental results and the continuous lines represent the analytical solutions. In the inset, the experimental and theoretical predictions are directly compared over the entire range of parameters being investigated in this work.

is more appropriate than the hydraulic diameter for nondimensionalizing the laminar continuum flow data of varied sections, the above results may further be extended to other cross-sectional shapes as well. To achieve that purpose, the product of friction factor and Reynolds number for circular sections may first be recast using $\sqrt{A}$ as the reference length scale to obtain

$$
f \operatorname{Re}_{\sqrt{A}}=\frac{8 \sqrt{\pi}}{1+4 \beta^{\prime}}+(f \operatorname{Re})_{\text {short }},
$$

so that

$$
(f \mathrm{Re})_{\text {short }}=\frac{2}{\sqrt{\pi}}\left[\frac{1}{3 \xi\left(1+4 \beta^{\prime}\right)^{2}}-2 \sum_{i=1}^{\infty} \frac{\left[3-\exp \left(-\pi \alpha_{i}^{2} \xi\right)\right] \exp \left(-\pi \alpha_{i}^{2} \xi\right)}{\alpha_{i}^{2} \xi\left[1+4 \beta^{\prime}+\alpha_{i}^{2} \beta^{\prime 2}\right]}\right],
$$

where $\xi=x / \sqrt{A} \operatorname{Re}_{\sqrt{A}}$. The term $(f \operatorname{Re})_{\text {short }}$ corresponds to a short duct asymptote $(\xi<0.001)$. For long microchannels, on the other hand, the "shape-dependent" frictional characteristics can be described as

$$
(f \text { Re })_{\text {long }}=\frac{12}{\left(1+11.97-10.59 \varepsilon+8.49 \varepsilon^{2}-2.11 \varepsilon^{3}\right) \frac{\beta^{\prime}}{2} \sqrt{\varepsilon}(1+\varepsilon)\left[1-\frac{192 \varepsilon}{\pi^{5}} \tanh \left(\frac{\pi}{2 \varepsilon}\right)\right]},
$$

where $\varepsilon$ is the aspect ratio (aspect ratio of all singly connected ducts is defined as the ratio of maximum width to maximum length of the section and is constrained to lie between 0 to 1 ). Derivation of the above equation is a straight forward extension of those presented by Muzychka and Yovanovich $^{30}$ toward the physical situation addressed in the present work. As per their definition, the aspect ratio for regular polygons is unity.

Based on the expressions for the long and the short duct asymptotes, a general model may be proposed by using the asymptotic correlation method proposed by Churchill and Usagi. ${ }^{31}$ The model takes the form 
TABLE I. Key results for rectangular and circular sectioned microconduits.

\begin{tabular}{llc}
\hline \hline & Rectangular section & Circular section \\
\hline Eigen condition & $\tan \alpha_{i}=\frac{\alpha_{i}}{1+\beta^{\prime} \alpha_{i}^{2}}$ & $\alpha_{i} J_{0}\left(\alpha_{i}\right)-2\left(1+0.5 \alpha_{i}^{2} \beta^{\prime}\right) J_{1}\left(\alpha_{i}\right)=0$ \\
$\begin{array}{l}\text { Velocity } \\
\text { profile }\end{array}$ & $V(\eta, \xi)=\frac{6 \beta^{\prime}}{6 \beta^{\prime}+2}+\frac{3\left(1-\eta^{2}\right)}{6 \beta^{\prime}+2}$ & $V(\eta, \xi)=\frac{4 \beta^{\prime}}{4 \beta^{\prime}+1}+\frac{2\left(1-\eta^{2}\right)}{4 \beta^{\prime}+1}+\sum_{i=1}^{\infty} \frac{4\left(1+0.5 \alpha_{i}^{2} \beta^{\prime}\right)}{\alpha_{i}\left[1+4 \beta^{\prime}+\alpha_{i}^{2} \beta^{\prime 2}\right]}$ \\
& $+\sum_{i=1}^{\infty} \frac{2\left[\alpha_{i} \cos \left(\alpha_{i} \eta\right)-\sin \left(\alpha_{i}\right)\right] \exp \left(-16 \alpha_{i}^{2} \xi\right)}{\alpha_{i}^{2} \sin \left(\alpha_{i}\right)\left[1+3 \beta^{\prime}+\alpha_{i}^{2} \beta^{\prime 2}\right]}$ & $+\left[\frac{J_{0}\left(\alpha_{i} \eta\right)}{J_{0}\left(\alpha_{i}\right)}-\frac{2}{2+\alpha_{i}^{2} \beta^{\prime}}\right] \exp \left(-4 \alpha_{i}^{2} \xi\right)$ \\
$\begin{array}{l}\begin{array}{l}\text { Friction } \\
\text { factor } \\
\left(f \text { Re } D_{h}\right)\end{array} \\
\begin{array}{l}\text { Fitted } \\
\text { entrance } \\
\text { length }\end{array}\end{array}$ & $\frac{24}{1+3 \beta^{\prime}}+\frac{1}{5 \xi\left(1+3 \beta^{\prime}\right)^{2}}-\sum_{i=1}^{\infty} \frac{\left[3-\exp \left(-16 \alpha_{i}^{2} \xi\right)\right] \exp \left(-16 \alpha_{i}^{2} \xi\right)}{\alpha_{i}^{2} \xi\left[1+3 \beta^{\prime}+\alpha_{i}^{2} \beta^{\prime 2}\right]}$ & $\frac{16}{1+4 \beta^{\prime}}+\frac{1}{3 \xi\left(1+4 \beta^{\prime}\right)^{2}}-2 \sum_{i=1}^{\infty} \frac{\left[3-\exp \left(-4 \alpha_{i}^{2} \xi\right)\right] \exp \left(-4 \alpha_{i}^{2} \xi\right)}{\alpha_{i}^{2} \xi\left[1+4 \beta^{\prime}+\alpha_{i}^{2} \beta^{\prime 2}\right]}$ \\
\hline \hline
\end{tabular}

$$
f \operatorname{Re}=\left[(f \mathrm{Re})_{\text {short }}^{n}+(f \mathrm{Re})_{\text {long }}^{n}\right]^{1 / n},
$$

where $n$ is a superposition parameter determined by comparison with the theoretical and experimental data over the full range of $\xi$ (the value of $n$ is ascertained by minimizing the root mean square deviation between the approximate solution and full-scale numerical solution). From the results obtained for the present analysis for rectangular and circular sections, a typical range of $n$ is obtained as $1<n<1.2$. Therefore, for approximate engineering analysis toward microfluidic system design, a value of $n$ close to unity may be taken without much loss of accuracy.

\section{CONCLUSIONS}

An analytical model is developed is this work to characterize the effects of hydrophobic interactions adjacent to the microchannel walls on the entrance region transport. However, additional interesting entrance and exit effects may occur due to electro-osmotic and/or electroviscous effects in narrow fluidic confinements, the details of which are reported elsewhere. $^{32,33}$ The present analytical model is first comprehensively validated with full-scale numerical simulations as well as comprehensive experimental investigations. The effects of hydrophobic interactions are considered to give rise to the formation of nanobubbles or a depleted lower-density wall-adjacent layer, according to the findings of the earlier researchers. Although results corresponding to simple geometries are only reported for the sake of brevity, trivial extensions can be made for other simple or complicated geometries, by appealing to the geometrical implication of an cross area-based length scale and a characteristic aspect ratio. From the present study, the following important observations can be made.

(i) With the formation of a depleted wall-adjacent layer of a less-dense fluid, the entrance length may get enhanced by a limit in the tune of $50 \%$. This enhancement does not magnify to indefinite proportions, however, because of competing mechanisms dictating the rate of shear layer growth. On the other hand, the enhancement effect is much more dramatic for nanobubble layers with relative thicknesses as low as of the order of 0.001 .

(ii) With thicker nanobubble layers, the flow velocities and accelerations in the central core region get substantially reduced in strength, at the cost of less significant disparities in the flow field between the walladjacent layer and the bulk region.

(iii) With thicker nanobubble layers (relative thicknesses greater than approximately 0.05), the friction factor characteristics exhibit negligible differences between developing and fully developed states, although for thinner nanobubble layers appreciable pressure drops are observed to occur in the developing region. Since typical nanobubble layer thicknesses (approximately in nanometers) are roughly three orders less than the characteristic microchannel dimensions (approximately in microns), the later case represents a technologically more interesting situation. With substantially enhanced entrance lengths being encountered for such cases, the corresponding friction factor predictions made by utilizing the present theory can be of immense practical consequence in designing microfluidic devices and systems with strong hydrophobic interactions. Inferences drawn from the present study clearly suggest that the implications of entrance region transport cannot be trivially overruled in such situations, especially for relatively shorter microchannels.

${ }^{1}$ T. Bayraktar and S. B. Pidugu, "Characterization of liquid flows in microfluidic systems," Int. J. Heat Mass Transfer 49, 815 (2006).

${ }^{2}$ R. J. Yang, L. M. Fu, and C. C. Hwang, "Electroosmotic entry flow in a microchannel," J. Colloid Interface Sci. 244, 173 (2001).

${ }^{3}$ X. Y. Chen, K. C. Toh, J. C. Chai, and C. Yang, "Developing pressuredriven liquid flow in microchannels under the electrokinetic effect," Int. J. Heat Mass Transfer 42, 609 (2004).

${ }^{4}$ L. A. Campbell and S. G. Kandlikar, "Effect of entrance condition on frictional losses and transition to turbulence in conventional and minichannel flows," Therm. Sci. Eng. 12, 1 (2004).

${ }^{5}$ C. Neto, D. R. Evans, E. Bonaccurso, H.-J. Butt, and V. S. J. Craig, "Boundary slip in Newtonian liquids: A review of experimental studies," Rep. Prog. Phys. 68, 2859 (2005). 
${ }^{6} \mathrm{~S}$. Chakraborty, "Order parameter modeling of fluid dynamics in narrow confinements subjected to hydrophobic interactions," Phys. Rev. Lett. 99, 094504 (2007).

${ }^{7}$ D. C. Tretheway and C. D. Meinhart, "A generating mechanism for apparent fluid slip in hydrophobic microchannels," Phys. Fluids 16, 1509 (2004).

${ }^{8}$ E. Lauga and M. P. Brenner, "Dynamic mechanisms for apparent slip on hydrophobic surfaces," Phys. Rev. E 70, 026311 (2004).

${ }^{9}$ R. Benzi, L. Biferale, M. Sbragaglia, S. Succi, and F. Toschi, "Mesoscopic two-phase model for describing apparent slip in microchannel flows," Europhys. Lett. 74, 651 (2006).

${ }^{10}$ M. Sbragaglia, R. Benzi, L. Biferale, S. Succi, and F. Toschi, "Surface roughness-hydrophobicity coupling in microchannel and nanochannel flows," Phys. Rev. Lett. 97, 204503 (2006)

${ }^{11}$ A. Steinberger, "High friction on a bubble mattress," Nat. Mater. 6, 665 (2007).

${ }^{12}$ P. G. de Gennes, "On fluid/wall slippage," Langmuir 18, 3413 (2002).

${ }^{13}$ W. L. Ryan and E. A. Hemmingsen, "Bubble formation at porous hydrophobic surfaces," J. Colloid Interface Sci. 197, 101 (1998).

${ }^{14} \mathrm{~S}$. G. Bankoff, "Entrainment of gas in the spreading of liquid over a rough surface," AIChE J. 4, 24 (1958).

${ }^{15}$ J. Tyrell and P. Attard, "Images of nanobubbles on hydrophobic surfaces and their interactions," Phys. Rev. Lett. 87, 176104 (2001).

${ }^{16}$ C. Cottin-Bizonne, J. L. Barrat, L. Boequet, and E. Charlaix, "Lowfriction flows of liquid at nanopatterned interfaces," Nat. Mater. 2, 237 (2003).

${ }^{17}$ R. K. Shah and A. L. London, Laminar Flow Forced Convection in Ducts (Academic, New York, 1978).

${ }^{18}$ E. Ruckenstein and P. Rajora, "On the no-slip boundary condition of hydrodynamics," J. Colloid Interface Sci. 96, 488 (1983).

${ }^{19}$ K. Lum, D. Chandler, and J. D. Weeks, "Hydrophobicity at small and large length scales," J. Phys. Chem. B 103, 4570 (1999).

${ }^{20} \mathrm{~S}$. Chakraborty, "Generalization of interfacial electrohydrodynamics in the presence of hydrophobic interactions in narrow fluidic confinements,"
Phys. Rev. Lett. 100, 097801 (2008).

${ }^{21}$ S. Chakraborty, "Towards a generalized representation of surface effects on pressure-driven liquid flow in microchannels," Appl. Phys. Lett. 90, 034108 (2007).

${ }^{22}$ S. V. Patankar, Numerical Heat Transfer and Fluid Flow (Hemisphere/ McGrawHill, Washington, 1980).

${ }^{23}$ P. de Gennes, F. Brochard-Wyart, and D. Quéré, Capillarity and Wetting

Phenomena: Drops, Bubbles, Pearls, Waves (Springer, New York, 2003).

${ }^{24}$ J. Bico, C. Marzolin, and D. Quéré, "Pearl drops," Europhys. Lett. 47, 220 (1999).

${ }^{25}$ J. Bico, C. Tordeux, and D. Quéré, "Rough wetting," Europhys. Lett. 55, 214 (2001)

${ }^{26}$ J. Bico, U. Thiele, and D. Quéré, "Wetting of textured surfaces," Colloids Surf., A 206, 41 (2002).

${ }^{27}$ D. Quéré, A. Lafuma, and J. Bico, "Slippy and sticky microtextured structures," Nanotechnology 14, 1109 (2003).

${ }^{28}$ S. Chakraborty, T. Das, and S. Chattoraj, "A generalized model for probing frictional characteristics of pressure-driven liquid microflows," J. Appl. Phys. 102, 104907 (2007).

${ }^{29}$ D. Sinton, "Microscale flow visualization," Microfluid. Nanofluid. 1, 2 (2004).

${ }^{30}$ Y. S. Muzychka and M. M. Yovanovich, "Laminar forced convection heat transfer in the combined entry region of non-circular ducts," J. Heat Transfer 126, 54 (2004).

${ }^{31} \mathrm{~S}$. W. Churchill and R. Usagi, "A general expression for the correlation of rates of transfer and other phenomena," Am. Inst. Chem. Eng. Symp. Ser. 18, 1121 (1972).

${ }^{32}$ S. Chakraborty and S. Padhy, "Induced pressure gradients due to entrance and exit effects in electroosmotically driven flows through nanopores within the continuum regime," J. Phys. D 41, 065502 (2008).

${ }^{33}$ S. Chakraborty and S. Das, "Streaming-field-induced convective transport and its influence on the electroviscous effects in narrow fluidic confinements beyond the Debye-Hückel limits," Phys. Rev. E 77, 037303 (2008). 